\title{
Certain inequalities associated with Hadamard $k$-fractional integral operators
}

Muharrem Tomar ${ }^{1}$, Shahid Mubeen ${ }^{2}$ and Junesang Choi ${ }^{3^{*}}$

\section{"Correspondence:}

junesang@dongguk.ac.kr

${ }^{3}$ Department of Mathematics,

Dongguk University, Gyeongju,

Republic of Korea

Full list of author information is

available at the end of the article

\begin{abstract}
We aim to present some new Pólya-Szegö type inequalities associated with Hadamard $k$-fractional integral operators, which are also used to derive some Chebyshev type integral inequalities. Further we apply some of the results presented here to a function which is bounded by the Heaviside functions.
\end{abstract}

MSC: 26A33;26D10;26D15

\section{Introduction and preliminaries}

We begin by recalling the following Chebyshev functional which has been investigated by many authors (see, e.g., [1-4]):

$$
T(f, g ; a, b)=\frac{1}{b-a} \int_{a}^{b} f(x) g(x) \mathrm{d} x-\frac{1}{(b-a)^{2}} \int_{a}^{b} f(x) \mathrm{d} x \int_{a}^{b} g(x) \mathrm{d} x,
$$

where $f, g:[a, b] \rightarrow \mathbb{R}$ are integrable functions on $[a, b]$. Here and in the following, let $\mathbb{R}$ and $\mathbb{R}^{+}$be the set of real and positive real numbers, respectively, and $\mathbb{R}_{0}^{+}:=\mathbb{R}^{+} \cup\{0\}$. Under more conditions $n \leq f(x) \leq N$ and $m \leq g(x) \leq M$ for all $x \in[a, b]$, where $n, m, N, M$ are real constants, the Chebyshev functional (1.1) satisfies the following inequality, which is known as Grüss integral inequality (see [5]; see also [6], p.236):

$$
|T(f, g ; a, b)| \leq \frac{1}{4}(M-m)(N-n)
$$

where the constant $\frac{1}{4}$ is sharp. In fact, the equality in (1.2) holds, for example, by taking

$$
f(x)=g(x)=\operatorname{sgn} x-\frac{a+b}{2} \quad(x \in[a, b])
$$

The Grüss inequality (1.2) has been investigated a lot and a number of its generalizations have been presented (see, e.g., [7-10]).

Let $f$ and $g$ be two positive integrable functions on $[a, b]$ such that

$$
0<m \leq f(x) \leq M<\infty \quad \text { and } \quad 0<n \leq f(x) \leq N<\infty .
$$

\section{空 Springer}

(c) 2016 Tomar et al. This article is distributed under the terms of the Creative Commons Attribution 4.0 International License (http://creativecommons.org/licenses/by/4.0/), which permits unrestricted use, distribution, and reproduction in any medium, provided you give appropriate credit to the original author(s) and the source, provide a link to the Creative Commons license, and indicate if changes were made. 
Pólya and Szegö [11] established the following inequality:

$$
\frac{\int_{a}^{b} f^{2}(x) \mathrm{d} x \int_{a}^{b} g^{2}(x) \mathrm{d} x}{\left(\int_{a}^{b} f(x) g(x) \mathrm{d} x\right)^{2}} \leq \frac{1}{4}\left(\sqrt{\frac{M N}{m n}}+\sqrt{\frac{m n}{M N}}\right)^{2},
$$

which was used by Dragomir and Diamond [12] who proved the following inequality:

$$
|T(f, g ; a, b)| \leq \frac{(M-m)(N-n)}{4(b-a)^{2} \sqrt{m M n N}} \int_{a}^{b} f(x) \mathrm{d} x \int_{a}^{b} g(x) \mathrm{d} x .
$$

Fractional calculus is a very helpful tool to perform differentiation and integration of real or complex number orders. This subject has earned much attention from researchers and mathematicians during the last few decades (see, e.g., [13-21]). Among a large number of the fractional integral operators developed, due to applications in many fields of sciences, the Riemann-Liouville fractional integral operator and Hadamard fractional integral operator have been extensively investigated.

Let $f \in L[a, b]$. Then the left-sided and the right-sided Hadamard fractional integrals of order $\alpha \geq 0$ and $a>0$ are defined, respectively, by

$$
H_{a^{+}}^{\alpha} f(t)=\frac{1}{\Gamma(\alpha)} \int_{a}^{t}\left(\ln \frac{t}{\tau}\right)^{\alpha-1} f(\tau) \frac{\mathrm{d} \tau}{\tau} \quad(0<a<t \leq b)
$$

and

$$
H_{b^{-}}^{\alpha} f(t)=\frac{1}{\Gamma(\alpha)} \int_{t}^{b}\left(\ln \frac{\tau}{t}\right)^{\alpha-1} f(\tau) \frac{\mathrm{d} \tau}{\tau} \quad(0<a \leq t<b) .
$$

The theory of $k$-functions has been investigated since, about a decade ago, Diaz and Pariguan [22] introduced the following generalizations of the classical gamma and beta functions, with a new parameter $k \in \mathbb{R}^{+}$, which are called $k$-gamma and $k$-beta functions, respectively:

$$
\Gamma_{k}(\alpha)=\int_{0}^{\infty} t^{\alpha-1} e^{-\frac{t^{k}}{k}} \mathrm{~d} t \quad(\Re(\alpha)>0)
$$

and

$$
B_{k}(\alpha, \beta)=\frac{1}{k} \int_{0}^{1} t^{\frac{\alpha}{k}-1}(1-t)^{\frac{\beta}{k}-1} \mathrm{~d} t \quad(\min \{\Re(\alpha), \Re(\beta)\}>0) .
$$

The functions $\Gamma_{k}$ defined on $\mathbb{R}^{+}$and $B_{k}(x, y)$ on $(0,1)$ satisfy the following properties:

(1) $\Gamma_{k}(x+k)=x \Gamma_{k}(x)$;

(2) $\Gamma_{k}(k)=1$;

(3) $\Gamma_{k}(x)$ is logarithmically convex;

(4) $B_{k}(x, y)=\frac{\Gamma_{k}(x) \Gamma_{k}(y)}{\Gamma_{k}(x+y)}$.

During the past several years, certain interesting properties, identities, and inequalities involving $k$-functions have been presented (see, e.g., [23-29]). Mubeen and Habibullah [30] used the $k$-gamma function $\Gamma_{k}(1.7)$ to introduce the following Riemann-Liouville 
type $k$-fractional integral:

$$
I_{a, k}^{\alpha} f(t)=\frac{1}{k \Gamma_{k}(\alpha)} \int_{a}^{t}(t-x)^{\frac{\alpha}{k}-1} f(x) \mathrm{d} x \quad(t \in[a, b]) .
$$

Later, Romero et al. [31] also used the $k$-gamma function $\Gamma_{k}$ (1.7) to introduce the $k$ Riemann-Liouville fractional derivative whose properties including a relationship with the $k$-fractional integral (1.9) were presented.

Using the $k$-gamma function with the parameter $k$, Mubeen et al. [32] have introduced left-sided and right-sided Hadamard $k$-fractional integrals of order $\alpha \in \mathbb{R}^{+}$, respectively, as follows: For $f \in L[a, b]$ and $k, a \in \mathbb{R}^{+}$,

$$
\mathcal{H}_{a^{+}, k}^{\alpha}\{f\}(t)=\frac{1}{k \Gamma_{k}(\alpha)} \int_{a}^{t}\left(\ln \frac{t}{\tau}\right)^{\frac{\alpha}{k}-1} f(\tau) \frac{\mathrm{d} \tau}{\tau} \quad(0<a<t \leq b)
$$

and

$$
\mathcal{H}_{b^{-}, k}^{\alpha}\{f\}(t)=\frac{1}{k \Gamma_{k}(\alpha)} \int_{t}^{b}\left(\ln \frac{\tau}{t}\right)^{\frac{\alpha}{k}-1} f(\tau) \frac{\mathrm{d} \tau}{\tau} \quad(0<a \leq t<b) .
$$

Using the Hadamard $k$-fractional integral and Proposition 6 in [22], we have

$$
\mathcal{H}_{a^{+}, k}^{\alpha}\{1\}(t)=\frac{(\ln (t / a))^{\frac{\alpha}{k}}}{\Gamma_{k}(\alpha+k)} \quad\left(0<a<t \leq b ; k, \alpha \in \mathbb{R}^{+}\right)
$$

and

$$
\mathcal{H}_{1^{+}, k}^{\alpha}\{1\}(t)=\frac{(\ln (t))^{\frac{\alpha}{k}}}{\Gamma_{k}(\alpha+k)} \quad\left(1<t \leq b ; k, \alpha \in \mathbb{R}^{+}\right) .
$$

\section{Some Pólya-Szegö and Chebyshev type inequalities involving the Hadamard k-fractional integrals}

In this section, we derive some new Pólya-Szegö type inequalities associated with the Hadamard $k$-fractional integral operators which are also used to establish some Chebyshev type integral inequalities.

Lemma 2.1 Let $f$ and $g$ be two positive real integrable functions defined on $[a, \infty)$. Also let $\varphi_{1}, \varphi_{2}, \psi_{1}$, and $\psi_{2}$ be integrable functions on $[a, \infty)$ such that

$$
0<\varphi_{1}(\tau) \leq f(\tau) \leq \varphi_{2}(\tau) \text { and } \quad 0<\psi_{1}(\tau) \leq g(\tau) \leq \psi_{2}(\tau)
$$

for all $\tau \in[a, t](t>a)$. Then, for $k, \alpha \in \mathbb{R}^{+}$, and $a \in \mathbb{R}_{0}^{+}$, the following inequality holds true:

$$
\frac{\mathcal{H}_{a^{+}, k}^{\alpha}\left\{\psi_{1} \psi_{2} f^{2}\right\}(t) \mathcal{H}_{a^{+}, k}^{\alpha}\left\{\varphi_{1} \varphi_{2} g^{2}\right\}(t)}{\left(\mathcal{H}_{a^{+}, k}^{\alpha}\left\{\left(\varphi_{1} \psi_{1}+\varphi_{2} \psi_{2}\right) f g\right\}(t)\right)^{2}} \leq \frac{1}{4}
$$

Proof Under the given conditions, we find

$$
\frac{f(\tau)}{g(\tau)} \leq \frac{\varphi_{2}(\tau)}{\psi_{1}(\tau)} \quad \text { and } \quad \frac{\varphi_{1}(\tau)}{\psi_{2}(\tau)} \leq \frac{f(\tau)}{g(\tau)} \quad(\tau \in[a, t](t>a))
$$


from which we have

$$
\left(\frac{\varphi_{2}(\tau)}{\psi_{1}(\tau)}-\frac{f(\tau)}{g(\tau)}\right)\left(\frac{f(\tau)}{g(\tau)}-\frac{\varphi_{1}(\tau)}{\psi_{2}(\tau)}\right) \geq 0
$$

and so

$$
\left(\frac{\varphi_{2}(\tau)}{\psi_{1}(\tau)}+\frac{\varphi_{1}(\tau)}{\psi_{2}(\tau)}\right) \frac{f(\tau)}{g(\tau)} \geq \frac{f^{2}(\tau)}{g^{2}(\tau)}+\frac{\varphi_{1}(\tau) \varphi_{2}(\tau)}{\psi_{1}(\tau) \psi_{2}(\tau)} .
$$

The inequality (2.4) can also be written as follows:

$$
\left(\varphi_{1}(\tau) \psi_{1}(\tau)+\varphi_{2}(\tau) \psi_{2}(\tau)\right) f(\tau) g(\tau) \geq \psi_{1}(\tau) \psi_{2}(\tau) f^{2}(\tau)+\varphi_{1}(\tau) \varphi_{2}(\tau) g^{2}(\tau)
$$

Here, multiplying each side of the inequality (2.5) by the following non-negative factor:

$$
\frac{1}{k \Gamma_{k}(\alpha)}\left(\ln \frac{t}{\tau}\right)^{\frac{\alpha}{k}-1} \frac{1}{\tau} \quad(\tau \in[a, t](t>a))
$$

and integrating the resulting inequality with respective to $\tau$ on $[a, t]$, we obtain

$$
\mathcal{H}_{a^{+}, k}^{\alpha}\left\{\left(\varphi_{1} \psi_{1}+\varphi_{2} \psi_{2}\right) f g\right\}(t) \geq \mathcal{H}_{a^{+}, k}^{\alpha}\left\{\psi_{1} \psi_{2} f^{2}\right\}(t)+\mathcal{H}_{a^{+}, k}^{\alpha}\left\{\varphi_{1} \varphi_{2} g^{2}\right\}(t) .
$$

Applying the AM-GM (the arithmetic-geometric mean) inequality,

$$
a+b \geq 2 \sqrt{a b} \quad\left(a, b \in \mathbb{R}_{0}^{+}\right)
$$

to the right-hand side of (2.6), we have

$$
\mathcal{H}_{a^{+}, k}^{\alpha}\left\{\left(\varphi_{1} \psi_{1}+\varphi_{2} \psi_{2}\right) f g\right\}(t) \geq 2 \sqrt{\mathcal{H}_{a^{+}, k}^{\alpha}\left\{\psi_{1} \psi_{2} f^{2}\right\}(t) \mathcal{H}_{a^{+}, k}^{\alpha}\left\{\varphi_{1} \varphi_{2} g^{2}\right\}(t)},
$$

which leads to

$$
\mathcal{H}_{a^{+}, k}^{\alpha}\left\{\psi_{1} \psi_{2} f^{2}\right\}(t) \mathcal{H}_{a^{+}, k}^{\alpha}\left\{\varphi_{1} \varphi_{2} g^{2}\right\}(t) \leq \frac{1}{4}\left(\mathcal{H}_{a^{+}, k}^{\alpha}\left\{\left(\varphi_{1} \psi_{1}+\varphi_{2} \psi_{2}\right) f g\right\}(t)\right)^{2} .
$$

This completes the proof.

The following corollary is easily seen to be a special case of Lemma 2.1 .

Corollary 1 Let $f$ and $g$ be two real positive integrable functions defined on $[a, \infty)$ such that

$$
0<m \leq f(\tau) \leq M<\infty \quad \text { and } \quad 0<n \leq g(\tau) \leq N<\infty \quad(\tau \in[a, t](t>a))
$$

where $n, N, m, M$ are real constants. Then, for all $t, k \in \mathbb{R}^{+}$and $\alpha \in \mathbb{R}^{+}$, we have

$$
\frac{\mathcal{H}_{a^{+}, k}^{\alpha}\left\{f^{2}\right\}(t) \mathcal{H}_{a^{+}, k}^{\alpha}\left\{g^{2}\right\}(t)}{\left(\mathcal{H}_{a^{+}, k}^{\alpha}\{f g\}(t)\right)^{2}} \leq \frac{1}{4}\left(\sqrt{\frac{m n}{M N}}+\sqrt{\frac{M N}{n m}}\right)^{2}
$$


Lemma 2.2 Let $f$ and $g$ be two positive real integrable functions defined on $[a, \infty)$. Also let $\varphi_{1}, \varphi_{2}, \psi_{1}$, and $\psi_{2}$ be integrable functions on $[a, \infty)$ satisfying the condition (2.1). Then, for $t>a\left(a \in \mathbb{R}_{0}^{+}\right)$and $k, \alpha, \beta \in \mathbb{R}^{+}$, the following inequality holds true:

$$
\frac{\mathcal{H}_{a^{+}, k}^{\alpha}\left\{\varphi_{1} \varphi_{2}\right\}(t) \mathcal{H}_{a^{+}, k}^{\beta}\left\{\psi_{1} \psi_{2}\right\}(t) \mathcal{H}_{a^{+}, k}^{\alpha}\left\{f^{2}\right\}(t) \mathcal{H}_{a^{+}, k}^{\beta}\left\{g^{2}\right\}(t)}{\left(\mathcal{H}_{a^{+}, k}^{\alpha}\left\{\varphi_{1} f\right\}(t) \mathcal{H}_{a^{+}, k}^{\beta}\left\{\psi_{1} g\right\}(t)+\mathcal{H}_{a^{+}, k}^{\alpha}\left\{\varphi_{2} f\right\}(t) \mathcal{H}_{a^{+}, k}^{\beta}\left\{\psi_{2} g\right\}(t)\right)^{2}} \leq \frac{1}{4} .
$$

Proof We find from (2.1) that

$$
\left(\frac{\varphi_{2}(\tau)}{\psi_{1}(\rho)}-\frac{f(\tau)}{g(\rho)}\right) \geq 0 \quad \text { and } \quad\left(\frac{f(\tau)}{g(\rho)}-\frac{\varphi_{1}(\tau)}{\psi_{2}(\rho)}\right) \geq 0 \quad(\tau, \rho \in[a, t](t>a))
$$

which yields

$$
\left(\frac{\varphi_{1}(\tau)}{\psi_{2}(\rho)}+\frac{\varphi_{2}(\tau)}{\psi_{1}(\rho)}\right) \frac{f(\tau)}{g(\rho)} \geq \frac{f^{2}(\tau)}{g^{2}(\rho)}+\frac{\varphi_{1}(\tau) \varphi_{2}(\tau)}{\psi_{1}(\rho) \psi_{2}(\rho)} .
$$

Multiplying each side of the inequality $(2.12)$ by $\psi_{1}(\rho) \psi_{2}(\rho) g^{2}(\rho)$, we get

$$
\varphi_{1}(\tau) f(\tau) \psi_{1}(\rho) g(\rho)+\varphi_{2}(\tau) f(\tau) \psi_{2}(\rho) g(\rho) \geq \psi_{1}(\rho) \psi_{2}(\rho) f^{2}(\tau)+\varphi_{1}(\tau) \varphi_{2}(\tau) g^{2}(\rho)
$$

Again multiplying each side of the inequality (2.13) by the following non-negative factor:

$$
\frac{1}{k^{2} \Gamma_{k}(\alpha) \Gamma_{k}(\beta)}\left(\ln \frac{t}{\tau}\right)^{\frac{\alpha}{k}-1}\left(\ln \frac{t}{\rho}\right)^{\frac{\beta}{k}-1} \frac{1}{\tau \rho} \quad(\tau, \rho \in[a, t](t>a))
$$

and integrating the resulting inequality with respect to $\tau$ and $\rho$ on $[a, t]$, we have

$$
\begin{aligned}
& \mathcal{H}_{a^{+}, k}^{\alpha}\left\{\varphi_{1} f\right\}(t) \mathcal{H}_{a^{+}, k}^{\beta}\left\{\psi_{1} g\right\}(t)+\mathcal{H}_{a^{+}, k}^{\alpha}\left\{\varphi_{2} f\right\}(t) \mathcal{H}_{a^{+}, k}^{\beta}\left\{\psi_{2} g\right\}(t) \\
& \geq \mathcal{H}_{a^{+}, k}^{\alpha}\left\{f^{2}\right\}(t) \mathcal{H}_{a^{+}, k}^{\beta}\left\{\psi_{1} \psi_{2}\right\}(t)+\mathcal{H}_{a^{+}, k}^{\beta}\left\{\varphi_{1} \varphi_{2}\right\}(t) \mathcal{H}_{a^{+}, k}^{\alpha}\left\{g^{2}\right\}(t) .
\end{aligned}
$$

Applying the AM-GM inequality (2.7) to (2.14), we obtain

$$
\begin{aligned}
& \mathcal{H}_{a^{+}, k}^{\alpha}\left\{\varphi_{1} f\right\}(t) \mathcal{H}_{a^{+}, k}^{\beta}\left\{\psi_{1} g\right\}(t)+\mathcal{H}_{a^{+}, k}^{\alpha}\left\{\varphi_{2} f\right\}(t) \mathcal{H}_{a^{+}, k}^{\beta}\left\{\psi_{2} g\right\}(t) \\
& \quad \geq 2 \sqrt{\mathcal{H}_{a^{+}, k}^{\alpha}\left\{f^{2}\right\}(t) \mathcal{H}_{a^{+}, k}^{\beta}\left\{\psi_{1} \psi_{2}\right\}(t) \mathcal{H}_{a^{+}, k}^{\alpha}\left\{\varphi_{1} \varphi_{2}\right\}(t) \mathcal{H}_{a^{+}, k}^{\beta}\left\{g^{2}\right\}(t)},
\end{aligned}
$$

which is easily seen to yield the desired inequality (2.11). Hence the proof is complete.

Corollary 2 Let $f$ and $g$ be two positive integrable functions on interval $[a, \infty)$ satisfying the conditions in (2.9). Then, for $t>1$ and $k, \alpha, \beta \in \mathbb{R}^{+}$, we have

$$
\frac{(\ln t)^{\frac{\alpha+\beta}{k}}}{\Gamma_{k}(\alpha+k) \Gamma_{k}(\beta+k)} \frac{\mathcal{H}_{1^{+}, k}^{\alpha}\left\{f^{2}(t)\right\} \mathcal{H}_{1^{+}, k}^{\beta}\left\{g^{2}(t)\right\}}{\left(\mathcal{H}_{1^{+}, k}^{\alpha}\{f(t)\}+\mathcal{H}_{1^{+}, k}^{\beta}\{g(t)\}\right)^{2}} \leq \frac{1}{4}\left(\sqrt{\frac{m n}{M N}}+\sqrt{\frac{M N}{m n}}\right)^{2} .
$$

Lemma 2.3 Suppose that all assumptions of Lemma 2.2 are satisfied. Then, for $t>a$ and $\alpha, \beta \in \mathbb{R}^{+}$, the following inequality holds true:

$$
\mathcal{H}_{a^{+}, k}^{\alpha}\left\{f^{2}\right\}(t) \mathcal{H}_{a^{+}, k}^{\beta}\left\{g^{2}\right\}(t) \leq \mathcal{H}_{a^{+}, k}^{\alpha}\left\{\left(\varphi_{2} f g\right) / \psi_{1}\right\}(t) \mathcal{H}_{a^{+}, k}^{\beta}\left\{\left(\psi_{2} f g\right) / \varphi_{1}\right\}(t) .
$$


Proof Using the conditions (2.1), we get

$$
\frac{1}{k \Gamma_{k}(\alpha)} \int_{a}^{t}\left(\ln \frac{t}{\tau}\right)^{\frac{\alpha}{k}-1} f^{2}(\tau) \frac{\mathrm{d} \tau}{\tau} \leq \frac{1}{k \Gamma_{k}(\alpha)} \int_{a}^{t}\left(\ln \frac{t}{\tau}\right)^{\frac{\alpha}{k}-1} \frac{\varphi_{2}(\tau)}{\psi_{1}(\tau)} f(\tau) g(\tau) \frac{\mathrm{d} \tau}{\tau}
$$

which implies

$$
\mathcal{H}_{a^{+}, k}^{\alpha}\left\{f^{2}\right\}(t) \leq \mathcal{H}_{a^{+}, k}^{\alpha}\left\{\left(\varphi_{2} f g\right) / \psi_{1}\right\}(t) .
$$

Similarly we have

$$
\frac{1}{k \Gamma_{k}(\beta)} \int_{a}^{t}\left(\ln \frac{t}{\rho}\right)^{\frac{\beta}{k}-1} g^{2}(\rho) \frac{\mathrm{d} \rho}{\rho} \leq \frac{1}{k \Gamma_{k}(\beta)} \int_{a}^{t}\left(\ln \frac{t}{\rho}\right)^{\frac{\beta}{k}-1} \frac{\psi_{2}(\rho)}{\varphi_{1}(\rho)} f(\rho) g(\rho) \frac{\mathrm{d} \rho}{\rho}
$$

and so

$$
\mathcal{H}_{a^{+}, k}^{\beta}\left\{g^{2}\right\}(t) \leq \mathcal{H}_{a^{+}, k}^{\beta}\left\{\left(\psi_{2} f g\right) / \varphi_{1}\right\}(t) .
$$

Multiplying the inequalities (2.18) and (2.19) side by side and considering all the involved terms are non-negative real numbers, we obtain the desired inequality (2.17).

It is easy to see from Lemma 2.3 that the assertion in Corollary 3 holds true.

Corollary 3 Let $f$ and $g$ be two positive integrable functions on interval $[a, \infty)$ satisfying the conditions in (2.9). Then, for $t>a$ and $\alpha, \beta \in \mathbb{R}^{+}$, we have

$$
\frac{\mathcal{H}_{a^{+}, k}^{\alpha}\left\{f^{2}\right\}(t) \mathcal{H}_{a^{+}, k}^{\beta}\left\{g^{2}\right\}(t)}{\mathcal{H}_{a^{+}, k}^{\alpha}\{f g\}(t) \mathcal{H}_{a^{+}, k}^{\beta}\{f g\}(t)} \leq \frac{M N}{m n} .
$$

Theorem 1 Let $f$ and $g$ be two positive integrable functions on interval $[a, \infty)$. Suppose that there exist four positive functions $\varphi_{1}, \varphi_{2}, \psi_{1}$, and $\psi_{2}$ satisfying the conditions (2.1). Then,for $t>a$ and $k, \alpha, \beta \in \mathbb{R}^{+}$, the following inequality holds true:

$$
\begin{aligned}
& \mid \frac{(\ln (t / a))^{\frac{\beta}{k}}}{\Gamma_{k}(\beta+k)} \mathcal{H}_{a^{+}, k}^{\alpha}\{f g\}(t)+\frac{(\ln (t / a))^{\frac{\alpha}{k}}}{\Gamma_{k}(\alpha+k)} \mathcal{H}_{a^{+}, k}^{\beta}\{f g\}(t) \\
& \quad-\mathcal{H}_{a^{+}, k}^{\alpha}\{f\}(t) \mathcal{H}_{a^{+}, k}^{\beta}\{g\}(t)-\mathcal{H}_{a^{+}, k}^{\alpha}\{g\}(t) \mathcal{H}_{a^{+}, k}^{\beta}\{f\}(t) \mid \\
& \leq\left|\mathrm{M}_{1}\left(f, \varphi_{1}, \varphi_{2}\right)(t)+\mathrm{M}_{2}\left(f, \varphi_{1}, \varphi_{2}\right)(t)\right|^{\frac{1}{2}} \\
& \quad \times\left|\mathrm{M}_{1}\left(g, \psi_{1}, \psi_{2}\right)(t)+\mathrm{M}_{2}\left(g, \psi_{1}, \psi_{2}\right)(t)\right|^{\frac{1}{2}},
\end{aligned}
$$

where

$$
\mathrm{M}_{1}(u, v, w)(t):=\frac{(\ln (t / a))^{\frac{\beta}{k}}}{4 \Gamma_{k}(\beta+k)} \frac{\left(\mathcal{H}_{a^{+}, k}^{\alpha}\{(v+w) u\}(t)\right)^{2}}{\mathcal{H}_{a^{+}, k}^{\alpha}\{v w\}(t)}-\mathcal{H}_{a^{+}, k}^{\alpha}\{u\}(t) \mathcal{H}_{a^{+}, k}^{\beta}\{u\}(t)
$$


and

$$
\mathrm{M}_{2}(u, v, w)(t):=\frac{(\ln (t / a))^{\frac{\alpha}{k}}}{4 \Gamma_{k}(\alpha+k)} \frac{\left(\mathcal{H}_{a^{+}, k}^{\beta}\{(v+w) u\}(t)\right)^{2}}{\mathcal{H}_{a^{+}, k}^{\beta}\{v w\}(t)}-\mathcal{H}_{a^{+}, k}^{\alpha}\{u\}(t) \mathcal{H}_{a^{+}, k}^{\beta}\{u\}(t)
$$

Proof Let

$$
H(\tau, \rho):=(f(\tau)-f(\rho))(g(\tau)-g(\rho))
$$

or, equivalently,

$$
H(\tau, \rho)=f(\tau) g(\tau)+f(\rho) g(\rho)-f(\tau) g(\rho)-f(\rho) g(\tau) .
$$

Upon multiplying each side of (2.22) by

$$
\frac{1}{k^{2} \Gamma_{k}(\alpha) \Gamma_{k}(\beta)}\left(\ln \frac{x}{\tau}\right)^{\frac{\alpha}{k}-1}\left(\ln \frac{x}{\rho}\right)^{\frac{\beta}{k}-1} \frac{1}{\tau \rho}
$$

and integrating the resulting identity with respect to $\tau$ and $\rho$ on $[a, t]$, we get

$$
\begin{gathered}
\frac{1}{k^{2} \Gamma_{k}(\alpha) \Gamma_{k}(\beta)} \int_{a}^{t} \int_{a}^{t}\left(\ln \frac{t}{\tau}\right)^{\frac{\alpha}{k}-1}\left(\ln \frac{t}{\rho}\right)^{\frac{\beta}{k}-1} H(\tau, \rho) \frac{\mathrm{d} \tau}{\tau} \frac{\mathrm{d} \rho}{\rho} \\
=\frac{(\ln (t / a))^{\frac{\beta}{k}}}{\Gamma_{k}(\beta+k)} \mathcal{H}_{a^{+}, k}^{\alpha}\{f g\}(t)+\frac{(\ln (t / a))^{\frac{\alpha}{k}}}{\Gamma_{k}(\alpha+k)} \mathcal{H}_{a^{+}, k}^{\beta}\{f g\}(t) \\
-\mathcal{H}_{a^{+}, k}^{\alpha}\{f\}(t) \mathcal{H}_{a^{+}, k}^{\beta}\{g\}(t)-\mathcal{H}_{a^{+}, k}^{\beta}\{f\}(t) \mathcal{H}_{a^{+}, k}^{\alpha}\{g\}(t) .
\end{gathered}
$$

Making use of the weighted Cauchy-Schwarz inequality for double integrals in (2.23), we have

$$
\begin{aligned}
&\left|\frac{1}{k^{2} \Gamma_{k}(\alpha) \Gamma_{k}(\beta)} \int_{a}^{t} \int_{a}^{t}\left(\ln \frac{t}{\tau}\right)^{\frac{\alpha}{k}-1}\left(\ln \frac{t}{\rho}\right)^{\frac{\beta}{k}-1} H(\tau, \rho) \frac{\mathrm{d} \tau}{\tau} \frac{\mathrm{d} \rho}{\rho}\right| \\
& \leq {\left[\frac{1}{k^{2} \Gamma_{k}(\alpha) \Gamma_{k}(\beta)} \int_{a}^{t} \int_{a}^{t}\left(\ln \frac{t}{\tau}\right)^{\frac{\alpha}{k}-1}\left(\ln \frac{t}{\rho}\right)^{\frac{\beta}{k}-1} f^{2}(\tau) \frac{\mathrm{d} \tau}{\tau} \frac{\mathrm{d} \rho}{\rho}\right.} \\
&+\frac{1}{k^{2} \Gamma_{k}(\alpha) \Gamma_{k}(\beta)} \int_{a}^{t} \int_{a}^{t}\left(\ln \frac{t}{\tau}\right)^{\frac{\alpha}{k}-1}\left(\ln \frac{t}{\rho}\right)^{\frac{\beta}{k}-1} f^{2}(\rho) \frac{d \tau}{\tau} \frac{\mathrm{d} \rho}{\rho} \\
&\left.-\frac{2}{k^{2} \Gamma_{k}(\alpha) \Gamma_{k}(\beta)} \int_{a}^{t} \int_{a}^{t}\left(\ln \frac{t}{\tau}\right)^{\frac{\alpha}{k}-1}\left(\ln \frac{t}{\rho}\right)^{\frac{\beta}{k}-1} f(\tau) f(\rho) \frac{d \tau}{\tau} \frac{\mathrm{d} \rho}{\rho}\right]^{\frac{1}{2}} \\
& \times\left[\frac{1}{k^{2} \Gamma_{k}(\alpha) \Gamma_{k}(\beta)} \int_{a}^{t} \int_{a}^{t}\left(\ln \frac{t}{\tau}\right)^{\frac{\alpha}{k}-1}\left(\ln \frac{t}{\rho}\right)^{\frac{\beta}{k}-1} g^{2}(\tau) \frac{\mathrm{d} \tau}{\tau} \frac{\mathrm{d} \rho}{\rho}\right. \\
&+\frac{1}{k^{2} \Gamma_{k}(\alpha) \Gamma_{k}(\beta)} \int_{a}^{t} \int_{a}^{t}\left(\ln \frac{t}{\tau}\right)^{\frac{\alpha}{k}-1}\left(\ln \frac{t}{\rho}\right)^{\frac{\beta}{k}-1} g^{2}(\rho) \frac{\mathrm{d} \tau}{\tau} \frac{\mathrm{d} \rho}{\rho} \\
&-\frac{2}{k^{2} \Gamma_{k}(\alpha) \Gamma_{k}(\beta)} \int_{a}^{t} \int_{a}^{t}\left(\ln \frac{t}{\tau}\right)^{\frac{\alpha}{k}-1}\left(\ln \frac{t}{\rho}\right)^{\frac{\beta}{k}-1} g(\tau) g(\rho) \frac{\mathrm{d} \frac{\mathrm{d} \rho}{\tau} \frac{{ }^{2}}{\rho}}{\frac{1}{2}}
\end{aligned}
$$


Then, upon using the Hadamard $k$-fractional integrals, we get

$$
\begin{aligned}
\mid & \frac{1}{k^{2} \Gamma_{k}(\alpha) \Gamma_{k}(\beta)} \int_{a}^{t} \int_{a}^{t}\left(\ln \frac{t}{\tau}\right)^{\frac{\alpha}{k}-1}\left(\ln \frac{t}{\rho}\right)^{\frac{\beta}{k}-1} H(\tau, \rho) \frac{\mathrm{d} \tau}{\tau} \frac{\mathrm{d} \rho}{\rho} \mid \\
\leq & {\left[\frac{(\ln (t / a))^{\frac{\beta}{k}}}{\Gamma_{k}(\beta+k)} \mathcal{H}_{a^{+}, k}^{\alpha}\left\{f^{2}\right\}(t)+\frac{(\ln (t / a))^{\frac{\alpha}{k}}}{\Gamma_{k}(\alpha+k)} \mathcal{H}_{a^{+}, k}^{\beta}\left\{f^{2}\right\}(t)-2 \mathcal{H}_{a^{+}, k}^{\alpha}\{f\}(t) \mathcal{H}_{a^{+}, k}^{\beta}\{f\}(t)\right]^{\frac{1}{2}} } \\
\times & {\left[\frac{(\ln (t / a))^{\frac{\beta}{k}}}{\Gamma_{k}(\beta+k)} \mathcal{H}_{a^{+}, k}^{\alpha}\left\{g^{2}\right\}(t)+\frac{(\ln (t / a))^{\frac{\alpha}{k}}}{\Gamma_{k}(\alpha+k)} \mathcal{H}_{a^{+}, k}^{\beta}\left\{g^{2}\right\}(t)\right.} \\
- & \left.2 \mathcal{H}_{a^{+}, k}^{\alpha}\{g\}(t) \mathcal{H}_{a^{+}, k}^{\beta}\{g\}(t)\right]^{\frac{1}{2}} .
\end{aligned}
$$

Setting $\psi_{1}(t)=\psi_{2}(t)=g(t)=1$ in Lemma 2.1, we obtain

$$
\frac{(\ln (t / a))^{\frac{\beta}{k}}}{\Gamma_{k}(\beta+k)} \mathcal{H}_{a^{+}, k}^{\alpha}\left\{f^{2}\right\}(t) \leq \frac{(\ln (t / a))^{\frac{\beta}{k}}}{4 \Gamma_{k}(\beta+k)} \frac{\left(\mathcal{H}_{a^{+}, k}^{\alpha}\left\{\left(\varphi_{1}+\varphi_{2}\right) f\right\}(t)\right)^{2}}{\mathcal{H}_{a^{+}, k}^{\alpha}\left\{\varphi_{1} \varphi_{2}\right\}(t)},
$$

which leads to

$$
\begin{aligned}
& \frac{(\ln (t / a))^{\frac{\beta}{k}}}{\Gamma_{k}(\beta+k)} \mathcal{H}_{a^{+}, k}^{\alpha}\left\{f^{2}\right\}(t)-\mathcal{H}_{a^{+}, k}^{\alpha}\{f\}(t) \mathcal{H}_{a^{+}, k}^{\beta}\{f\}(t) \\
& \quad \leq \frac{(\ln (t / a))^{\frac{\beta}{k}}}{4 \Gamma_{k}(\beta+k)} \frac{\left(\mathcal{H}_{a^{+}, k}^{\alpha}\left\{\left(\varphi_{1}+\varphi_{2}\right) f\right\}(t)\right)^{2}}{\mathcal{H}_{a^{+}, k}^{\alpha}\left\{\varphi_{1} \varphi_{2}\right\}(t)}-\mathcal{H}_{a^{+}, k}^{\alpha}\{f\}(t) \mathcal{H}_{a^{+}, k}^{\beta}\{f\}(t) \\
& \quad=\mathrm{M}_{1}\left(f, \varphi_{1}, \varphi_{2}\right)(t)
\end{aligned}
$$

and

$$
\begin{aligned}
& \frac{(\ln (t / a))^{\frac{\alpha}{k}}}{\Gamma_{k}(\alpha+k)} \mathcal{H}_{a^{+}, k}^{\beta}\left\{f^{2}\right\}(t)-\mathcal{H}_{a^{+}, k}^{\alpha}\{f\}(t) \mathcal{H}_{a^{+}, k}^{\beta}\{f\}(t) \\
& \quad \leq \frac{(\ln (t / a))^{\frac{\alpha}{k}}}{4 \Gamma_{k}(\alpha+k)} \frac{\left(\mathcal{H}_{a^{+}, k}^{\beta}\left\{\left(\varphi_{1}+\varphi_{2}\right) f\right\}(t)\right)^{2}}{\mathcal{H}_{a^{+}, k}^{\beta}\left\{\varphi_{1} \varphi_{2}\right\}(t)}-\mathcal{H}_{a^{+}, k}^{\alpha}\{f\}(t) \mathcal{H}_{a^{+}, k}^{\beta}\{f\}(t) \\
& \quad=\mathrm{M}_{2}\left(f, \varphi_{1}, \varphi_{2}\right)(t) .
\end{aligned}
$$

Similarly, taking $\varphi_{1}(t)=\varphi_{2}(t)=f(t)=1$ in Lemma 2.1, we get

$$
\frac{(\ln (t / a))^{\frac{\beta}{k}}}{\Gamma_{k}(\beta+k)} \mathcal{H}_{a^{+}, k}^{\alpha}\left\{g^{2}\right\}(t)-\mathcal{H}_{a^{+}, k}^{\alpha}\{g\}(t) \mathcal{H}_{a^{+}, k}^{\beta}\{g\}(t) \leq \mathrm{M}_{1}\left(g, \psi_{1}, \psi_{2}\right)(t)
$$

and

$$
\frac{(\ln (t / a))^{\frac{\alpha}{k}}}{\Gamma_{k}(\alpha+k)} \mathcal{H}_{a^{+}, k}^{\beta}\left\{g^{2}\right\}(t)-\mathcal{H}_{a^{+}, k}^{\alpha}\{g\}(t) \mathcal{H}_{a^{+}, k}^{\beta}\{g\}(t) \leq \mathrm{M}_{2}\left(g, \psi_{1}, \psi_{2}\right)(t) .
$$

Finally, by combining the inequalities (2.25)-(2.29), we can get the desired inequality (2.21). This completes the proof.

The following assertion is a special case of Theorem 1 when $\alpha=\beta$. 
Theorem 2 Suppose that the assumptions of Theorem 1 are satisfied. Then, for $t>1$ and $\alpha \in \mathbb{R}^{+}$, the following inequality holds true:

$$
\begin{aligned}
& \left|\frac{(\ln (t))^{\frac{\alpha}{k}}}{\Gamma_{k}(\alpha+k)} \mathcal{H}_{a^{+}, k}^{\alpha}\{f g\}(t)-\mathcal{H}_{a^{+}, k}^{\alpha}\{f\}(t) \mathcal{H}_{a^{+}, k}^{\alpha}\{g\}(t)\right| \\
& \quad \leq\left|\mathcal{M}\left(f, \varphi_{1}, \varphi_{2}\right)(t) \mathcal{M}\left(g, \varphi_{1}, \varphi_{2}\right)(t)\right|^{\frac{1}{2}}
\end{aligned}
$$

where

$$
\mathcal{M}(u, v, w)(t):=\frac{(\ln (t))^{\frac{\alpha}{k}}}{4 \Gamma_{k}(\alpha+k)} \frac{\left(\mathcal{H}_{a^{+}, k}^{\alpha}\{(v+w) u\}(t)\right)^{2}}{\mathcal{H}_{a^{+}, k}^{\alpha}\{v w\}(t)}-\left(\mathcal{H}_{a^{+}, k}^{\alpha}\{u\}(t)\right)^{2} .
$$

Remark 2.1 Setting $\varphi_{1}=m, \varphi_{2}=M, \psi_{1}=n$, and $\psi_{2}=N$, we have

$$
\mathcal{M}(f, m, M)(t)=\frac{(M-m)^{2}}{4 m M}\left(\mathcal{H}_{a^{+}, k}^{\alpha}\{f\}(t)\right)^{2}
$$

and

$$
\mathcal{M}(g, n, N)(t)=\frac{(N-n)^{2}}{4 n N}\left(\mathcal{H}_{a^{+}, k}^{\alpha}\{g\}(t)\right)^{2} .
$$

Corollary 4 Let $f$ and $g$ be two positive integrable functions on $[a, \infty)$ satisfying the condition (2.9). Then, for $t>a$ and $\alpha \in \mathbb{R}^{+}$, we have

$$
\begin{aligned}
& \left|\frac{(\ln (t / a))^{\frac{\alpha}{k}}}{\Gamma_{k}(\alpha+k)} \mathcal{H}_{a^{+}, k}^{\alpha}\{f g\}(t)-\mathcal{H}_{a^{+}, k}^{\alpha}\{f\}(t) \mathcal{H}_{a^{+}, k}^{\alpha}\{g\}(t)\right| \\
& \quad \leq \frac{(M-m)(N-n)}{4 \sqrt{m M n N}} \mathcal{H}_{a^{+}, k}^{\alpha}\{f\}(t) \mathcal{H}_{a^{+}, k}^{\alpha}\{g\}(t) .
\end{aligned}
$$

\section{Applications}

In this section we apply Hadamard $k$-fractional integrals to a function which is bounded by the Heaviside functions.

The simplest piecewise continuous function is the unit step function, which is known as the Heaviside function, defined by

$$
u_{c}(t)= \begin{cases}1 & \text { if } u \geq c \\ 0 & \text { if } u<c\end{cases}
$$

The unit step function is basically an on-off switch which is very useful in differential equations and piecewise functions when there is a large number of pieces, for example, Riemann sums as in Figure 1. Using Heaviside function, a piecewise continuous function $\varphi_{1}(t)$ defined on an interval $[a, T]$ can be written as follows:

$$
\begin{aligned}
\varphi_{1}(t) & =m_{1}\left(u_{t_{0}}(t)-u_{t_{1}}(t)\right)+m_{2}\left(u_{t_{1}}(t)-u_{t_{2}}(t)\right)+m_{3}\left(u_{t_{3}}(t)-u_{t_{2}}(t)\right)+\cdots+m_{p+1} u_{t_{p}}(t) \\
& =m_{1} u_{t_{0}}+\left(m_{2}-m_{1}\right) u_{t_{1}}(t)+\left(m_{3}-m_{2}\right) u_{t_{2}}(t)+\cdots+\left(m_{p+1}-m_{p}\right) u_{t_{p}}(t) \\
& =\sum_{k=0}^{p}\left(m_{k+1}-m_{k}\right) u_{t_{k}}(t),
\end{aligned}
$$




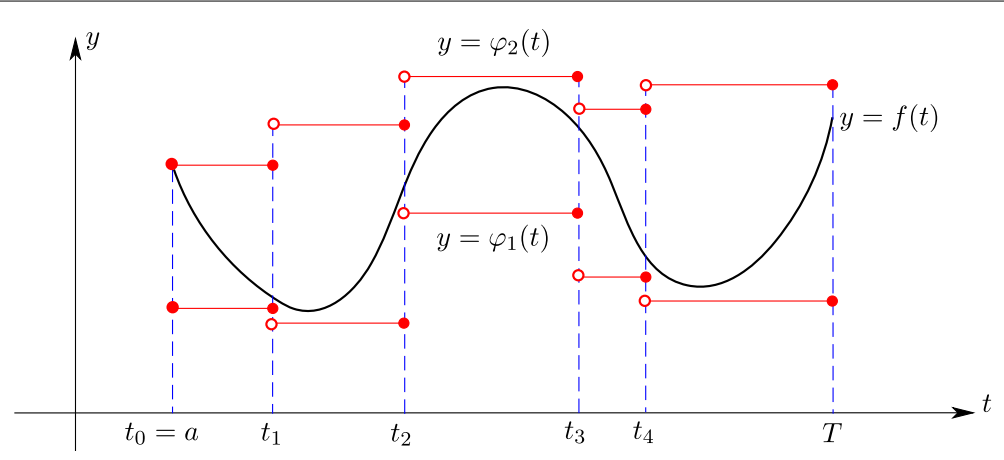

Figure 1 Functions $f, \varphi_{1}$, and $\varphi_{2}$.

where $m_{0}=0, m_{i} \in \mathbb{R}(i=0,1, \ldots, p+1)$ and $a=t_{0}<t_{1}<t_{2}<\cdots<t_{p}<t_{p+1}=T$. Similarly we define the functions $\varphi_{2}, \psi_{1}$, and $\psi_{2}$ as follows:

$$
\begin{aligned}
& \varphi_{2}(t)=\sum_{k=0}^{p}\left(M_{k+1}-M_{k}\right) u_{t_{k}}(t), \\
& \psi_{1}(t)=\sum_{k=0}^{p}\left(n_{k+1}-n_{k}\right) u_{t_{k}}(t), \\
& \psi_{2}(t)=\sum_{k=0}^{p}\left(N_{k+1}-N_{k}\right) u_{t_{k}}(t),
\end{aligned}
$$

where $n_{0}=N_{0}=M_{0}=0$ and $n_{i}, N_{i}, M_{i} \in \mathbb{R}(i=0,1, \ldots, p+1)$.

Let $f$ be an integrable function on $[a, T]$ which satisfies the condition (2.1) with the functions $\varphi_{1}, \varphi_{2}, \psi_{1}$, and $\psi_{2}$ in (3.1), (3.2), (3.3) and (3.4), respectively. Then we get $m_{j+1} \leq f(t) \leq M_{j+1}$ for each $t \in\left(t_{j}, t_{j+1}\right)(j=0,1, \ldots, p)$. For example, Figure 1 represents the case $p=4$.

Then the Hadamard $k$-fractional integral of $f$ on $[a, T]$ can be defined as follows:

$$
\mathcal{H}_{a^{+}, k}^{\alpha}\{f\}(T)=\sum_{j=0}^{p} \mathcal{H}_{t_{j, t}, t_{j+1}, k}^{\alpha}\{f\}(t),
$$

where

$$
\mathcal{H}_{t_{j}, t_{j+1}, k}^{\alpha}\{f\}(t):=\frac{1}{k \Gamma_{k}(\alpha)} \int_{t_{j}}^{t_{j+1}}\left(\ln \frac{t}{s}\right)^{\frac{\alpha}{k}-1} f(s) \frac{\mathrm{d} s}{s} \quad(j=0,1,2, \ldots, p) .
$$

Proposition 1 Let $f$ and $g$ be two positive integrable functions on $[a, T]$ which satisfy the condition (2.1) with the functions $\varphi_{1}, \varphi_{2}, \psi_{1}$, and $\psi_{2}$ in (3.1), (3.2), (3.3), and (3.4), respectively. Then, for $\alpha \in \mathbb{R}^{+}$, the following inequality holds true:

$$
\begin{aligned}
& \left(\sum_{j=0}^{p} n_{j+1} N_{j+1} \mathcal{H}_{t_{j}, t_{j+1}, k}^{\alpha}\left\{f^{2}\right\}(T)\right)\left(\sum_{j=0}^{p} m_{j+1} M_{j+1} \mathcal{H}_{t_{j}, t_{j+1}, k}^{\alpha}\left\{g^{2}\right\}(T)\right) \\
& \quad \leq \frac{1}{4} \sum_{j=0}^{p}\left(n_{j+1} N_{j+1}+m_{j+1} M_{j+1}\right)\left(\mathcal{H}_{a^{+}, k}^{\alpha}\{g\}(T)\right)^{2} .
\end{aligned}
$$


Proof Using the Hadamard $k$-fractional integral in (3.5), we get

$$
\begin{aligned}
& \mathcal{H}_{a^{+}, k}\left\{\psi_{1} \psi_{2} f^{2}\right\}(T)=\sum_{j=0}^{p} n_{j+1} N_{j+1} \mathcal{H}_{t_{j}, t_{j+1}, k}^{\alpha}\left\{f^{2}\right\}(T), \\
& \mathcal{H}_{a^{+}, k}\left\{\varphi_{1} \varphi_{2} g^{2}\right\}(T)=\sum_{j=0}^{p} m_{j+1} M_{j+1} \mathcal{H}_{t_{j}, t_{j+1}, k}^{\alpha}\left\{g^{2}\right\}(T),
\end{aligned}
$$

and

$$
\mathcal{H}_{a^{+}, k}\left\{\left(\varphi_{1} \psi_{1}+\varphi_{2} \psi_{2} f g\right)\right\}(T)=\sum_{j=0}^{p}\left(m_{j+1} n_{j+1}+M_{j+1} N_{j+1}\right) \mathcal{H}_{t_{j}, t_{j+1}, k}^{\alpha}\{f g\}(T)
$$

Then substituting equalities (3.8), (3.9), and (3.10) for the result in Lemma 2.1 yields the desired result (3.7).

Proposition 2 Suppose that assumptions of Proposition 1 are satisfied. Then, for $k, \alpha, \beta \in$ $\mathbb{R}^{+}$, we have

$$
\begin{aligned}
& \mid \frac{(\ln (t / a))^{\frac{\beta}{k}}}{\Gamma_{k}(\beta+k)} \mathcal{H}_{a^{+}, k}^{\alpha}\{f g\}(T)+\frac{(\ln (t / a))^{\frac{\alpha}{k}}}{\Gamma_{k}(\alpha+k)} \mathcal{H}_{a^{+}, k}^{\beta}\{f g\}(T) \\
& \quad-\mathcal{H}_{a^{+}, k}^{\alpha}\{f\}(t) \mathcal{H}_{a^{+}, k}^{\beta}\{g\}(T)-\mathcal{H}_{a^{+}, k}^{\beta}\{f\}(t) \mathcal{H}_{a^{+}, k}^{\alpha}\{f\}(T) \mid \\
& \leq\left|\mathrm{M}_{1}^{*}\left(f, m_{j+1}, M_{j+1}\right)(t)+\mathrm{M}_{2}^{*}\left(f, m_{j+1}, M_{j+1}\right)(T)\right|^{\frac{1}{2}} \\
& \quad \times\left|\mathrm{M}_{1}^{*}\left(g, n_{j+1}, N_{j+1}\right)(t)+\mathrm{M}_{2}^{*}\left(g, n_{j+1}, N_{j+1}\right)(T)\right|^{\frac{1}{2}},
\end{aligned}
$$

where

$$
\begin{aligned}
\mathrm{M}_{1}^{*}(u, v, w)(t):= & \frac{(\ln (t / a))^{\frac{\beta}{k}} \Gamma_{k}(\alpha+k)}{4 \Gamma_{k}(\beta+k)} \frac{\sum_{j=0}^{p}(v+w)\left(\mathcal{H}_{t_{j}, t_{j+1}, k}^{\alpha}\{u\}(t)\right)^{2}}{\sum_{j=0}^{p} v w\left[\left(\ln \left(t / t_{j}\right)\right]^{\frac{\alpha}{k}}-\left[\ln \left(t / t_{j+1}\right)\right)^{\frac{\alpha}{k}}\right]} \\
& -\left(\mathcal{H}_{a^{+}, k}^{\alpha}\{u\}(T)\right)\left(\mathcal{H}_{a^{+}, k}^{\beta}\{u\}(T)\right), \\
\mathrm{M}_{2}^{*}(u, v, w)(t):= & \frac{(\ln (t / a))^{\frac{\alpha}{k}} \Gamma_{k}(\beta+k)}{4 \Gamma_{k}(\alpha+k)} \frac{\sum_{j=0}^{p}(v+w)\left(\mathcal{H}_{t_{j}, t_{j+1}, k}^{\beta}\{u\}(t)\right)^{2}}{\sum_{j=0}^{p} v w\left[\left(\ln \left(t / t_{j}\right)\right]^{\frac{\beta}{k}}-\left[\ln \left(t / t_{j+1}\right)\right)^{\frac{\beta}{k}}\right]} \\
& -\left(\mathcal{H}_{a^{+}, k}^{\beta}\{u\}(t)\right)\left(\mathcal{H}_{a^{+}, k}^{\alpha}\{u\}(t)\right) .
\end{aligned}
$$

Proof Since

$$
\begin{aligned}
\mathcal{H}_{t_{j}, t_{j+1}, k}^{\alpha}\{f\}(T) & =\frac{1}{k \Gamma_{k}(\alpha)} \int_{t_{j}}^{t_{j+1}}\left(\ln \frac{t}{s}\right)^{\frac{\alpha}{k}-1} f(s) \frac{\mathrm{d} s}{s} \\
& =\frac{\left(\ln \left(t / t_{j}\right)\right)^{\frac{\alpha}{k}}-\left(\ln \left(t / t_{j+1}\right)\right)^{\frac{\alpha}{k}}}{\Gamma_{k}(\alpha+k)},
\end{aligned}
$$


we get

$$
\mathcal{H}_{a^{+}, k}\left\{\varphi_{1} \varphi_{2}\right\}\{T\}=\sum_{j=0}^{p} \frac{m_{j+1} M_{j+1}}{\Gamma_{k}(\alpha+k)}\left[\left(\ln \left(t / t_{j}\right)\right)^{\frac{\alpha}{k}}-\left(\ln \left(t / t_{j+1}\right)\right)^{\frac{\alpha}{k}}\right]
$$

and

$$
\mathcal{H}_{a^{+}, k}\left\{\psi_{1} \psi_{2}\right\}\{T\}=\sum_{j=0}^{p} \frac{n_{j+1} N_{j+1}}{\Gamma_{k}(\alpha+k)}\left[\left(\ln \left(t / t_{j}\right)\right)^{\frac{\alpha}{k}}-\left(\ln \left(t / t_{j+1}\right)\right)^{\frac{\alpha}{k}}\right] .
$$

After some computations, we have

$$
\begin{aligned}
\mathrm{M}_{1}\left(f, \varphi_{1}, \varphi_{2}\right)(T)= & \frac{(\ln (t / a))^{\frac{\beta}{k}} \Gamma_{k}(\alpha+k)}{4 \Gamma_{k}(\beta+k)} \frac{\sum_{j=0}^{p}\left(m_{j+1}+M_{j+1}\right)\left(\mathcal{H}_{t_{j}, t_{j+1}, k}^{\alpha}\{f\}(t)\right)^{2}}{\sum_{j=0}^{p} m_{j+1} M_{j+1}\left[\left(\ln \left(t / t_{j}\right)\right]^{\frac{\alpha}{k}}-\left[\ln \left(t / t_{j+1}\right)\right)^{\frac{\alpha}{k}}\right]} \\
& -\left(\mathcal{H}_{a^{+}, k}^{\alpha}\{f\}(T)\right)\left(\mathcal{H}_{a^{+}, k}^{\beta}\{f\}(T)\right), \\
\mathrm{M}_{1}\left(g, \psi_{1}, \psi_{2}\right)(T)= & \frac{(\ln (t / a))^{\frac{\beta}{k}} \Gamma_{k}(\alpha+k)}{4 \Gamma_{k}(\beta+k)} \frac{\sum_{j=0}^{p}\left(n_{j+1}+N_{j+1}\right)\left(\mathcal{H}_{t_{j}, t_{j+1}, k}^{\alpha}\{g\}(t)\right)^{2}}{\sum_{j=0}^{p} n_{j+1} N_{j+1}\left[\left(\ln \left(t / t_{j}\right)\right]^{\frac{\alpha}{k}}-\left[\ln \left(t / t_{j+1}\right)\right)^{\frac{\alpha}{k}}\right]} \\
& -\left(\mathcal{H}_{a^{+}, k}^{\alpha}\{g\}(T)\right)\left(\mathcal{H}_{a^{+}, k}^{\beta}\{g\}(T)\right), \\
\mathrm{M}_{2}\left(f, \varphi_{1}, \varphi_{2}\right)(T)= & \frac{(\ln (t / a))^{\frac{\alpha}{k}} \Gamma_{k}(\beta+k)}{4 \Gamma_{k}(\alpha+k)} \frac{\sum_{j=0}^{p}\left(m_{j+1}+M_{j+1}\right)\left(\mathcal{H}_{t_{j}, t_{j+1}, k}^{\beta}\{f\}(t)\right)^{2}}{\sum_{j=0}^{p} m_{j+1} M_{j+1}\left[\left(\ln \left(t / t_{j}\right)\right]^{\frac{\beta}{k}}-\left[\ln \left(t / t_{j+1}\right)\right)^{\frac{\beta}{k}}\right]} \\
& -\left(\mathcal{H}_{a^{+}, k}^{\alpha}\{g\}(T)\right)\left(\mathcal{H}_{a^{+}, k}^{\beta}\{g\}(T)\right),
\end{aligned}
$$

and

$$
\begin{aligned}
\mathrm{M}_{2}\left(g, \psi_{1}, \psi_{2}\right)(t)= & \frac{(\ln (t / a))^{\frac{\alpha}{k}} \Gamma_{k}(\beta+k)}{4 \Gamma_{k}(\alpha+k)} \frac{\sum_{j=0}^{p}\left(n_{j+1}+N_{j+1}\right)\left(\mathcal{H}_{t_{j}, t_{j+1}, k}^{\beta}\{g\}(T)\right)^{2}}{\sum_{j=0}^{p} n_{j+1} N_{j+1}\left[\left(\ln \left(t / t_{j}\right)\right]^{\frac{\beta}{k}}-\left[\ln \left(t / t_{j+1}\right)\right)^{\frac{\beta}{k}}\right]} \\
& -\left(\mathcal{H}_{a^{+}, k}^{\alpha}\{g\}(T)\right)\left(\mathcal{H}_{a^{+}, k}^{\beta}\{g\}(T)\right) .
\end{aligned}
$$

By applying the results here to Theorem 1, we obtain the desired inequality (3.11). Hence the proof is complete.

The special case of Proposition 2 when $\alpha=\beta$ is seen immediately to reduce to the result in Corollary 5.

Corollary 5 Suppose that the assumptions of Proposition 2 are satisfied. Then, for $k, \alpha \in$ $\mathbb{R}^{+}$, the following inequality holds true:

$$
\begin{aligned}
& \left|\frac{(\ln (t / a))^{\frac{\alpha}{k}}}{\Gamma_{k}(\alpha+k)} \mathcal{H}_{a^{+}, k}^{\alpha}\{f g\}(T)-\mathcal{H}_{a^{+}, k}^{\alpha}\{f\}(T) \mathcal{H}_{a^{+}, k}^{\alpha}\{f\}(T)\right| \\
& \quad \leq\left|\mathrm{M}^{*}\left(f, m_{j+1}, M_{j+1}\right)(T) \mathrm{M}^{*}\left(g, n_{j+1}, N_{j+1}\right)(T)\right|^{\frac{1}{2}},
\end{aligned}
$$


where

$$
\mathrm{M}^{*}(u, v, w)(t)=\frac{(\ln (t / a))^{\frac{\alpha}{k}}}{4} \frac{\sum_{j=0}^{p}(v+w)\left(\mathcal{H}_{t_{j}, t_{j+1}, k}^{\alpha}\{u\}(t)\right)^{2}}{\sum_{j=0}^{p} v w\left[\left(\ln \left(t / t_{j}\right)\right]^{\frac{\alpha}{k}}-\left[\ln \left(t / t_{j+1}\right)\right)^{\frac{\alpha}{k}}\right]}-\left(\mathcal{H}_{a^{+}, k}^{\alpha}\{u\}(t)\right)^{2} .
$$

We conclude this paper by remarking that all the results presented in this paper can be converted into those for the right-sided Hadamard $k$-fractional integral.

\section{Competing interests}

The authors declare that they have no competing interests.

\section{Authors' contributions}

The authors have contributed equally to this manuscript. They read and approved the final manuscript.

\section{Author details}

${ }^{1}$ Department of Mathematics, Faculty of Science and Arts, Ordu University, Ordu, Turkey. ${ }^{2}$ Department of Mathematics, University of Sargodha, Sargodha, Pakistan. ${ }^{3}$ Department of Mathematics, Dongguk University, Gyeongju, Republic of Korea.

\section{Acknowledgements}

This work was supported by Dongguk University Research Fund (Gyeongju). The authors would like to express their deep-felt thanks for the reviewers' helpful comments.

Received: 30 June 2016 Accepted: 18 September 2016 Published online: 26 September 2016

\section{References}

1. Cerone, P, Dragomir, SS: New upper and lower bounds for the Chebyshev functional. J. Inequal. Pure Appl. Math. 3(5), Article 77 (2002)

2. Dahmani, Z, Mechouar, O, Brahami, S: Certain inequalities related to the Chebyshev's functional involving a Riemann-Liouville operator. Bull. Math. Anal. Appl. 3(4), 38-44 (2011)

3. Pečarić, J, Perić, I: Identities for the Chebyshev functional involving derivatives of arbitrary order and applications. J. Math. Anal. Appl. 313, 475-483 (2006)

4. Purohit, SD, Kalla, SL: Certain inequalities related to the Chebyshev's functional involving Erdélyi-Kober operators. Scientia, Ser. A, Math. Sci. 25, 55-63 (2014)

5. Grüss, G: Über das maximum des absoluten betrages von $\frac{1}{b-a} \int_{a}^{b} f(x) g(x) d x-\frac{1}{(b-a)^{2}} \int_{a}^{b} f(x) d x \int_{a}^{b} g(x) d x$. Math. Z. 39, 215-226 (1935)

6. Mitrinović, DS, Pečarić, JE, Fink, AM: Classical and New Inequalities in Analysis. Kluwer Academic, Dordrecht (1993)

7. Dragomir, SS: A generalization of Grüss inequality in inner product spaces and applications. J. Math. Anal. Appl. 237, 74-82 (1999)

8. Elezovic, N, Marangunic, LJ, Pecaric, J: Some improvements of Grüss type inequality. J. Math. Inequal. 1, 425-436 (2007)

9. Pachpatte, BG: On multidimensional Grüss type inequalities. J. Inequal. Pure Appl. Math. 3(2), Article 27 (2002)

10. Li, X, Mohapatra, RN, Rodriguez, RS: Grüss-type inequalities. J. Math. Anal. Appl. 267(2), 434-443 (2002)

11. Pólya, G, Szegö, G: Aufgaben und Lehrsatze aus der Analysis, vol. 1. Die Grundlehren der mathmatischen Wissenschaften, vol. 19. Springer, Berlin (1925)

12. Dragomir, SS, Diamond, NT: Integral inequalities of Grüss type via Pólya-Szegö and Shisha-Mond results. East Asian Math. J. 19(1), 27-39 (2003)

13. Belarbi, S, Dahmani, Z: On some new fractional integral inequalities. J. Inequal. Pure Appl. Math. 10(3), Article 86 (2009)

14. Chebyshev, PL: Sur les expressions approximatives des integrales definies par les autres prises entre les mmes limites. Proc. Math. Soc. Charkov 2, 93-98 (1882)

15. Dahmani, Z: New inequalities in fractional integrals. Int. J. Nonlinear Sci. 9(4), 493-497 (2010)

16. Dahmani, Z, Tabharit, L: On weighted Grüss type inequalities via fractional integration. J. Adv. Res. Pure Math. 2, 31-38 (2010)

17. Kiryakova, V: Generalized Fractional Calculus and Applications. Pitman Res. Notes Math. Ser., vol. 301. Longman, New York (1994)

18. Ntouyas, KS, Agarwal, P, Tariboon, J: On Pólya-Szegö and Chebyshev types inequalities involving the Riemann-Liouville fractional integral operators. J. Math. Inequal. 10(2), 491-504 (2016)

19. Podlubny, I: Fractional Differential Equations. Academic Press, London (1999)

20. Sarıkaya, MZ, Dahmani, Z, Kiriş, ME, Ahmad, F: (k,s)-Riemann-Liouville fractional integral and applications. Hacet. J. Math. Stat. 45(1), 77-89 (2016)

21. Set, E, Tomar, M, Sarıkaya, MZ: On generalized Grüss type inequalities for k-fractional integrals. Appl. Math. Comput. 269, 29-34 (2015)

22. Diaz, R, Pariguan, E: On hypergeometric functions and Pochhammer k-symbol. Divulg. Mat. 15, 179-192 (2007)

23. Kokologiannaki, CG: Properties and inequalities of generalized k-gamma, beta and zeta functions. Int. J. Contemp. Math. Sci. 5(14), 653-660 (2010)

24. Kokologiannaki, CG, Karasniqi, V: Some properties of k-gamma function. Matematiche LXVIII, 13-22 (2013) 
25. Kokologiannaki, CG, Sourla, VD: Bounds for k-gamma and k-beta functions. J. Inequal. Spec. Funct. 4(3), 1-5 (2013)

26. Krasniqi, $\mathrm{V}$ : Inequalities and monotonicity for the ratio of $k$-gamma function. Sci. Magna 6, 40-45 (2010)

27. Krasniqi, V: A limit for the k-gamma and k-beta function. Int. Math. Forum 5, 1613-1617 (2010)

28. Suryanarayana, R, Rao, C: Some new inequalities for the generalized $\epsilon$-gamma, beta and zeta functions. Int. J. Eng. Res. Technol. 1(9), 1-4 (2012)

29. Zhang, J, Shi, HN: Two double inequalities for k-gamma and k-Riemann zeta functions. J. Inequal. Appl. 2014, 191 (2014)

30. Mubeen, S, Habibullah, GM: k-Fractional integrals and application. Int. J. Contemp. Math. Sci. 7, 89-94 (2012)

31. Romero, LG, Luque, LL, Dorrego, GA, Cerutti, RA: On the k-Riemann-Liouville fractional derivative. Int. J. Contemp. Math. Sci. 8(1), 41-51 (2013)

32. Mubeen, S, lqbal, S, Tomar, M: On Hadamard $k$-fractional integrals and applications (submitted)

Submit your manuscript to a SpringerOpen ${ }^{\bullet}$ journal and benefit from:

- Convenient online submission

- Rigorous peer review

- Immediate publication on acceptance

- Open access: articles freely available online

- High visibility within the field

- Retaining the copyright to your article 